\title{
Set and the encoding of visual stimuli
}

ELEANOR SEXTON AND JOHN P. MCLAUGHLIN UNIVERSITY OF DELA WARE

Encoding strategy and its consistency with method of report were varied to evaluate their interaction with the effect of set. Set instructions lowered errors for Ss encoding by separate stimulus dimensions, but not for Ss encoding by syntax. Syntax encoders made more errors for figures on the right than on the left. Set seems to affect the order in which information is encoded when the strategy permits such treatment.

It is generally acknowledged that instructions to attend to certain aspects of stimulus arrays facilitate $\mathrm{Ss}^{\top}$ reports of those aspects. There is disagreement, however, on the identity of the processes affected by the attention instructions (cf., Haber, 1966). The alternative general hypotheses have been that attention instructions, or set (a) enhance the actual perception of stimuli, or (b) affect the report rather than the percept itself.

Harris \& Haber (1963) have reported evidence which strongly suggests that set affects the order in which information is verbally encoded. Their Ss were trained to encode briefly presented stimuli with either a code based on English syntax, separately describing segregated aspects of a stimulus array, e.g., "two blue stars, three red triangles" (Sx code), or one which separately described each dimension of the total array, e.g., "two-three, blue-red, stars-triangles" (Dm code). Harris and Haber found that instructions to attend to one dimension of the total array, e.g., color, facilitated the report of that dimension for the Dm encoders but had no effect on the performance of the Sx encoders.

The generality of Harris \& Haber's (1963) results is limited by one factor, however. To prevent Ss from always reporting the emphasized dimension first, forced orders of report were used. To do this, Harris and Haber presented a set of response alternatives for each separate dimension, requiring Ss to choose among one set before going on to the next. Since all Ss were thus reporting by dimensions, the Ss using the Dm code were reporting in a manner consistent with their encoding strategy, but those using the Sx code were not. The absence of a facilitative effect of set in the performance of the Sx encoders may have been due to an encoding strategy-method of report inconsistency and not to an inefficient strategy alone. The purpose of the present experiment was to determine whether Sx encoders would show a facilitating effect of set when their method of report was consistent with their encoding strategy.

\section{Method}

Three male and 27 female students at the University of Delaware volunteered for the experiment. Each $S$ was tested individually.

The apparatus and procedure of the experiment were similar in many respects to those of Harris \& Haber
(1963). Stimuli were composed of two sets of figures on $13 \times 12 \mathrm{~cm}$ white cards. Each set contained one, two, three, or four figures which were uniform in color and shape. Figure colors were red, blue, yellow, or green, and the shapes were triangles, squares, circles, and stars. The two sets on a given card were different in the number, color, and shape of their figures. The longest dimension of a given shape subtended a visual angle of $5.4 \mathrm{deg}$. Figures were cut from construction paper and glued to the cards. Eighty stimuli were presented to $S$ in a tachistoscope (Lafayette Electro-Tach).

Two groups of $10 \mathrm{Ss}$ were trained to use the Sx code and one group the Dm code. Training in the Sx code consisted of four stimulus presentations with instructions to report all that $S$ had seen and four trials with attention instructions. After each trial $E$ gave the correct answer in the $\mathrm{Sx}$ code and after the fourth trial, E told $S$ "I think you will find it easiest if after each presentation you say out loud once what you have seen, e.g., two blue stars, three green triangles." Ss receiving training in the Dm code were shown a stimulus card and instructed to describe one dimension, identified by $\mathbf{E}$, who then gave the correct answer. After seven trials, E said "I think you will find it easiest if after each presentation you say aloud once what you have seen, keeping in mind to report by dimensions, e.g., blue-green, star-triangle. ${ }^{18}$ Six additional trials were given with attention instructions, which emphasized either color or shape, but never number. Ss were told that they would receive 30 points for correctly reporting the emphasized dimension, 3 points for the unemphasized dimension, and 3 points for reporting the number. They were encouraged to amass as many points as possible.

Two methods of report were used. Ten Sx encoders orally encoded and then wrote their descriptions in syntax (Group $\mathrm{Sx}-\mathrm{Sx}$ ). The remaining $10 \mathrm{Sx}$ encoders (Group Sx-Dm) and the $10 \mathrm{Dm}$ encoders (Group Dm$\mathrm{Dm}$ ) were required to report in dimensions. Ss were given check sheets listing the alternative shape and color terms and were instructed to respond after oral encoding by writing the number of perceived figures next to the appropriate terms. Ss were forced to begin at the top of each sheet and the terms for each dimension were alternated in their position on the page, thus controlling order of report.

Color and shape were each emphasized for 40 trials. Oral encoding and report immediately followed the stimulus presentation. The exposure duration was $100 \mathrm{msec}$.

Results

Table 1 summarizes the errors committed by each group in reporting color and shape, when those di- 
Table 1 Errors in reporting Emphasized (Em) and Unemphasized (UnEm) dimensions for three groups

\begin{tabular}{lccccccc}
\hline & Report & \multicolumn{5}{c}{ Groups } \\
Dimensions & Order & Em & UnEm & Em & UnEm & Em & UnEm \\
\hline Color & 1st & 19 & 15 & 8 & 8 & 3 & 10 \\
& 2nd & & & 7 & 8 & 12 & 7 \\
Shape & 1st & 41 & 34 & 18 & 11 & 11 & 14 \\
& 2nd & & & 16 & 13 & 21 & 14 \\
\hline
\end{tabular}

mensions were emphasized and unemphasized, for each order of report. Only errors in reporting shape and color were analyzed, since the report of number was never differentially emphasized in the instructions. A forced order of report was not used for Group Sx-Sx so that no effort was made to distinguish between errors for figures reported first or second. All errors for this group are recorded as errors in first report.

These data suggest that encoding strategy influenced the effectiveness of emphasis instructions. Only Group Dm-Dm tended to report emphasized dimensions with fewer errors than unemphasized dimensions, but this was significant only in the report of color in first report $\left(X^{2}=3.77, d f=1, p=053\right)$. Since the prediction was directional, this effect was considered significant with a one-tailed $\mathrm{p}=.027$. No effects of emphasis instructions were found for Groups $\mathrm{Sx}-\mathrm{Sx}$ and $\mathrm{Sx}-\mathrm{Dm}$, nor were these groups different from each other in proportion of errors for emphasized and unemphasized dimensions. Across all groups, the report of color was more accurate than the report of shape $\left(\chi^{2}=31.78\right.$, $\mathrm{df}=1, \mathrm{p}<.001$ ).

It was also found that errors in reporting figures on the left (L) of the displays were generally lower than errors for those on the right (R), but encoding strategy and method of report influenced the magnitude of the difference. Errors for $\mathrm{R}$ and $\mathrm{L}$, respectively, were: Group Sx-Sx, 81 and 37; Group Sx-Dm, 55 and 38; Group Dm-Dm, 50 and 43. The magnitude of difference $(\mathrm{R}-\mathrm{L})$ in Group $\mathrm{Sx}-\mathrm{Sx}$ is greater than in Group Sx-Dm $\left(x^{2}=11.08\right.$, df $\left.=1, p<.001\right)$, and the difference in Group $\mathrm{Sx}-\mathrm{Dm}$ is greater than that in Group Dm-Dm $\left(X^{2}=4.16, \mathrm{df}=1, \mathrm{p}<.05\right)$.

\section{Discussion}

The data of the present experiment are in general agreement with those of Harris \& Haber (1963) and support their contention that set affects the verbal encoding of the stimulus pattern. It seems that the Dm code permitted $\mathrm{Ss}$ to give preferential treatment to the emphasized dimension resulting in a facilitative effect of set, while the Sx code did not provide such an opportunity. The absence of any difference in error distribution between Groups Sx-Sx and Sx-Dm suggests that consistency of encoding strategy with method of report is not a relevant variable in this situation.

The reasons for the weakness of the set effect when reporting shapes for Group Dm-Dm are open to speculation. Since shape was more difficult than color in general, some competition may have resulted when shape was emphasized, leading to more errors. This differential difficulty between color and shape was not found by Harris \& Haber (1963). This may be due to a sex difference, since their study employed male Ss and most of the Ss in the present study were female. Female Ss may be more sensitive to color than to geometric forms.

The difference among the groups in the magnitude of the R-L error discrepancy indicates that each encoding strategy produces a characteristic error pattern, and raises the possibility that the facilitating effect of set is a function of an interaction between type of set and encoding strategy. The Dm code can provide an avenue for preferential treatment of an emphasized dimension, when dimensions are defined as they were in the present experiment, but one would expect that the $\mathrm{Sx}$ code would be the more appropriate for instructions emphasizing spatial divisions.

\section{References}

HABER, R. N. Nature of the effect of set on perception. Psychol. Rev., $1966,73,335-351$.

HARRIS, C. S., \& HABER, R. N. Selective attention and coding in visual perception. J. exp. Psychol., 1963, 65, 328-333.

Note

1. Based on a thesis submitted to the faculty of the University of Delaware by the senior author in partial fulfillment of the requirements for a B. A. with distinction. 